\title{
SOLUTION OF QUADRATIC NONLINEAR PROBLEMS WITH MULTIPLE SCALES LINDSTEDT-POINCARE METHOD
}

\author{
Mehmet Pakdemirli and Gözde Sarı \\ Applied Mathematics and Computation Center, Celal Bayar University, Muradiye, \\ 45140 Manisa, Turkey. \\ mpak@cbu.edu.tr, gozde.deger@cbu.edu.tr
}

\begin{abstract}
A recently developed perturbation algorithm namely the multiple scales Lindstedt-Poincare method (MSLP) is employed to solve the mathematical models. Three different models with quadratic nonlinearities are considered. Approximate solutions are obtained with classical multiple scales method (MS) and the MSLP method and they are compared with the numerical solutions. It is shown that MSLP solutions are better than the MS solutions for the strongly nonlinear case of the considered models.
\end{abstract}

Keywords- perturbation methods; numerical solutions; systems with quadratic nonlinearities

\section{INTRODUCTION}

Perturbation theories have been widely used to obtain approximate analytical solutions of linear and nonlinear physical problems. Although the methods provide acceptable solutions for weakly nonlinear problems, the solutions do not represent the physics for the strongly nonlinear cases. Recently, for solution of the strongly nonlinear problems, a new perturbation method was developed by Pakdemirli et al. [1]. This method named multiple scales Lindstedt-Poincare method (MSLP) combines the classical multiple scales method and the Lindstedt-Poincare method. Pakdemirli and Karahan [2] and Pakdemirli et al. [3] applied the method to many strongly nonlinear problems and obtained good results compatible with the numerical solutions.

This new method has not been tested for problems with strong quadratic nonlinearities. In this study, three different quadratic nonlinear problems are solved by MSLP and MS method. The approximate solutions are contrasted with the numerical solutions. For weak nonlinearities, all three methods yield similar solutions. As the nonlinearity is increased, the solutions deviate from each other, with MSLP yielding better approximate solutions in contrast to the numerical solutions.

\section{QUADRATIC NONLINEAR MODEL I}

Consider the below problem with a quadratic nonlinearity $\ddot{u}+\omega_{0}{ }^{2} u+\varepsilon u \dot{u}=0$

with initial conditions

$u(0)=a_{0} \quad \dot{u}(0)=0$ 


\section{1. Multiple Scales Method (MS)}

First, the problem is solved with the classical method. Solutions are assumed to be of the form;

$$
u=u_{0}\left(T_{0}, T_{1}, T_{2}\right)+\varepsilon u_{1}\left(T_{0}, T_{1}, T_{2}\right)+\varepsilon^{2} u_{2}\left(T_{0}, T_{1}, T_{2}\right)
$$

where $T_{0}=t$ is the usual fast time scale, $T_{1}=\varepsilon t$ and $T_{2}=\varepsilon^{2} t$ are the slow time scales. Time derivatives are defined as

$$
\frac{d}{d t}=D_{0}+\varepsilon D_{1}+\varepsilon^{2} D_{2}, \quad \frac{d^{2}}{d t^{2}}=D_{0}^{2}+2 \varepsilon D_{0} D_{1}+\varepsilon^{2}\left(D_{1}^{2}+2 D_{0} D_{2}\right)
$$

where $D_{n}=\partial / \partial T_{n}$.

If (3) and (4) are substituted into the original equation, the following equations are obtained at each order of $\varepsilon$;

$$
\begin{aligned}
& O(1) \quad D_{0}{ }^{2} u_{0}+\omega_{0}{ }^{2} u_{0}=0 \quad u_{0}(0)=a_{0} \quad D_{0} u_{0}(0)=0 \\
& \mathrm{O}(\varepsilon) \quad D_{0}{ }^{2} u_{1}+\omega_{0}{ }^{2} u_{1}=-2 D_{0} D_{1} u_{0}-u_{0} D_{0} u_{0} \quad u_{1}(0)=0 \quad\left(D_{0} u_{1}+D_{1} u_{0}\right)(0)=0 \\
& \mathrm{O}\left(\varepsilon^{2}\right) \quad D_{0}^{2} u_{2}+\omega_{0}^{2} u_{2}=-2 D_{0} D_{1} u_{1}-\left(D_{1}^{2}+2 D_{0} D_{2}\right) u_{0}-u_{0}\left(D_{0} u_{1}+D_{1} u_{0}\right)-u_{1} D_{0} u_{0}
\end{aligned}
$$

At order 1, the solution may be expressed as

$$
u_{0}=A e^{i \omega_{0} T_{0}}+c c
$$

where cc stands for the complex conjugates of the preceding terms and

$$
A=\frac{1}{2} a e^{i \beta}
$$

The first order solution is obtained in terms of real amplitude and phase

$$
u_{0}=a\left(T_{1}, T_{2}\right) \cos \left(\omega_{0} t+\beta\left(T_{1}, T_{2}\right)\right)
$$

Applying the initial conditions yields

$$
\beta(0)=0 \quad a(0)=a_{0}
$$

Equation (8) is substituted into (6) and secular terms are eliminated

$$
D_{1} A=0 \Rightarrow A=A\left(T_{2}\right)
$$

The solution at order $\varepsilon$ is

$$
u_{1}=B e^{i \omega_{0} T_{0}}+\frac{i}{3 \omega_{0}} A^{2} e^{2 i \omega_{0} T_{0}}+c c
$$

This solution may be represented in terms of real amplitude and phase

$$
u_{1}=-b \sin \left(\omega_{0} T_{0}+\gamma\right)-\frac{a^{2}}{6 \omega_{0}} \sin \left(2 \omega_{0} T_{0}+2 \beta\right)
$$

where

$$
B=\frac{1}{2} i b e^{i \gamma}
$$

Applying the initial conditions yields

$$
\gamma(0)=0 \quad b(0)=-\frac{a_{0}{ }^{2}}{3 \omega_{0}}
$$

Equation (8) and (13) are inserted into (7) and secular terms are eliminated,

$$
-2 i \omega_{0} D_{1} B-2 i \omega_{0} D_{2} A+\frac{1}{3} A^{2} \bar{A}=0
$$

If (5), (15), (16) are inserted into (17), one finally has

$$
a=a_{0}, \quad b=-\frac{a_{0}^{2}}{3 \omega_{0}}, \quad \gamma=0, \quad \beta=-\frac{a_{0}^{2}}{24 \omega_{0}} T_{2}
$$

The final solution is 


$$
u=a_{0} \cos \left(\left(\omega_{0}-\frac{\varepsilon^{2} a_{0}^{2}}{24 \omega_{0}}\right) t\right)+\frac{\varepsilon a_{0}^{2}}{6 \omega_{0}}\left(2 \sin \left(\omega_{0} t\right)-\sin \left(2\left(\omega_{0}-\frac{\varepsilon^{2} a_{0}^{2}}{24 \omega_{0}}\right) t\right)\right)
$$

For valid solutions, the correction term should be much smaller than the leading term. For the problem, this criterion yields

$$
\frac{\varepsilon a_{0}}{6 \omega_{0}}<<1
$$

\subsection{Multiple Scales Lindstedt-Poincare Method (MSLP)}

Details of the method was presented in the previous literature [1-3]. The time transformation $\tau=\omega t$ is applied to the model

$$
\omega^{2} u^{\prime \prime}+\omega_{0}^{2} u+\varepsilon u \omega u^{\prime}=0
$$

where prime denotes derivative with respect to the new variable $\tau$. The time scales in this method are slightly different from classical multiple scales

$$
T_{0}=\tau, T_{1}=\varepsilon \tau, T_{2}=\varepsilon^{2} \tau
$$

Expressing the time derivatives

$$
\frac{d}{d t}=D_{0}+\varepsilon D_{1}+\varepsilon^{2} D_{2} \quad \frac{d^{2}}{d t^{2}}=D_{0}^{2}+2 \varepsilon D_{0} D_{1}+\varepsilon^{2}\left(D_{1}^{2}+2 D_{0} D_{2}\right)
$$

with $\mathrm{D}_{\mathrm{n}}=\partial / \partial T_{n}$ and substituting the expansions

$$
\begin{aligned}
& u=u_{0}\left(T_{0}, T_{1}, T_{2}\right)+\varepsilon u_{1}\left(T_{0}, T_{1}, T_{2}\right)+\varepsilon^{2} u_{2}\left(T_{0}, T_{1}, T_{2}\right) \\
& \omega_{0}{ }^{2}=\omega^{2}-\varepsilon \omega_{1}-\varepsilon^{2} \omega_{2}
\end{aligned}
$$

into the original equation, the following equations are obtained at each order of approximation;

$$
\begin{aligned}
& O(1) \quad \omega^{2} D_{0}{ }^{2} u_{0}+\omega^{2} u_{0}=0, \quad u_{0}(0)=a_{0} \quad D_{0} u_{0}(0)=0 \\
& \mathrm{O}(\varepsilon) \quad \omega^{2} D_{0}{ }^{2} u_{1}+\omega^{2} u_{1}=-2 \omega^{2} D_{0} D_{1} u_{0}+\omega_{1} u_{0}-\omega u_{0} D_{0} u_{0} \quad u_{1}(0)=0 \quad\left(D_{0} u_{1}+D_{1} u_{0}\right)(0)=0 \\
& \mathrm{O}\left(\varepsilon^{2}\right) \quad \omega^{2} D_{0}{ }^{2} u_{2}+\omega^{2} u_{2}=-2 \omega^{2} D_{0} D_{1} u_{1}-\omega^{2}\left(D_{1}{ }^{2}+2 D_{0} D_{2}\right) u_{0}+\omega_{1} u_{1}+\omega_{2} u_{0} \\
&
\end{aligned}
$$

The first order solution is

$$
u_{0}=A e^{i T_{0}}+c c=a \cos \left(T_{0}+\beta\right)
$$

Applying the initial conditions yields

$$
\beta(0)=0, a(0)=a_{0}
$$

Equation (29) is substituted into (27) and secular terms are eliminated

$$
-2 i \omega^{2} D_{1} A+\omega_{1} A=0
$$

If $\mathrm{D}_{1} \mathrm{~A}=0$ is selected, $\mathrm{a}=\mathrm{a}\left(\mathrm{T}_{2}\right), \beta=\beta\left(\mathrm{T}_{2}\right)$ and $\omega_{1}=0$. Since $\omega_{1}$ is not complex, this choice is admissible. The solution at order $\varepsilon$ is

$$
u_{1}=B e^{i T_{0}}+\frac{i}{3 \omega} A^{2} e^{2 i T_{0}}+c c \quad B=\frac{1}{2} i b e^{i \gamma}
$$

In terms of real amplitude and phase, the solution is

$$
u_{1}=-b \sin \left(T_{0}+\gamma\right)-\frac{a^{2}}{6 \omega} \sin \left(2 T_{0}+2 \beta\right)
$$

Applying the initial conditions yields

$$
\gamma(0)=0 \quad b(0)=-\frac{a_{0}^{2}}{3 \omega}
$$

Equations (29) and (32) are inserted into (28) and secular terms are eliminated 
$-2 i \omega^{2} D_{1} B-2 i \omega^{2} D_{2} A+\omega_{2} A+\frac{1}{3} A^{2} \bar{A}=0$

$\mathrm{D}_{1} \mathrm{~B}=0$ can be assumed. If $\mathrm{D}_{2} \mathrm{~A}=0$ is selected, $\omega_{2}$ comes out to be real and this is again an admissible choice. After algebraic calculations, Equation (35) yields

$$
a=a_{0}, \quad b=-\frac{a_{0}^{2}}{3 \omega}, \quad \gamma=\beta=0, \quad \omega_{2}=-\frac{1}{12} a^{2}
$$

The frequency is

$$
\omega=\sqrt{\omega_{0}^{2}-\frac{\varepsilon^{2} a_{0}^{2}}{12}}
$$

The final solution in terms of this frequency is

$$
u=a_{0} \cos (\omega t)+\frac{\varepsilon a_{0}^{2}}{6 \omega}(2 \sin (\omega t)-\sin (2 \omega t))+O\left(\varepsilon^{2}\right)
$$

For valid solutions, the perturbation criteria is

$$
\frac{\varepsilon a_{0}}{6 \omega_{0} \sqrt{1-\frac{\varepsilon^{2}}{12 \omega_{0}^{2}} a_{0}^{2}}}<<1
$$

\subsection{Comparisons with the Numerical Solutions}

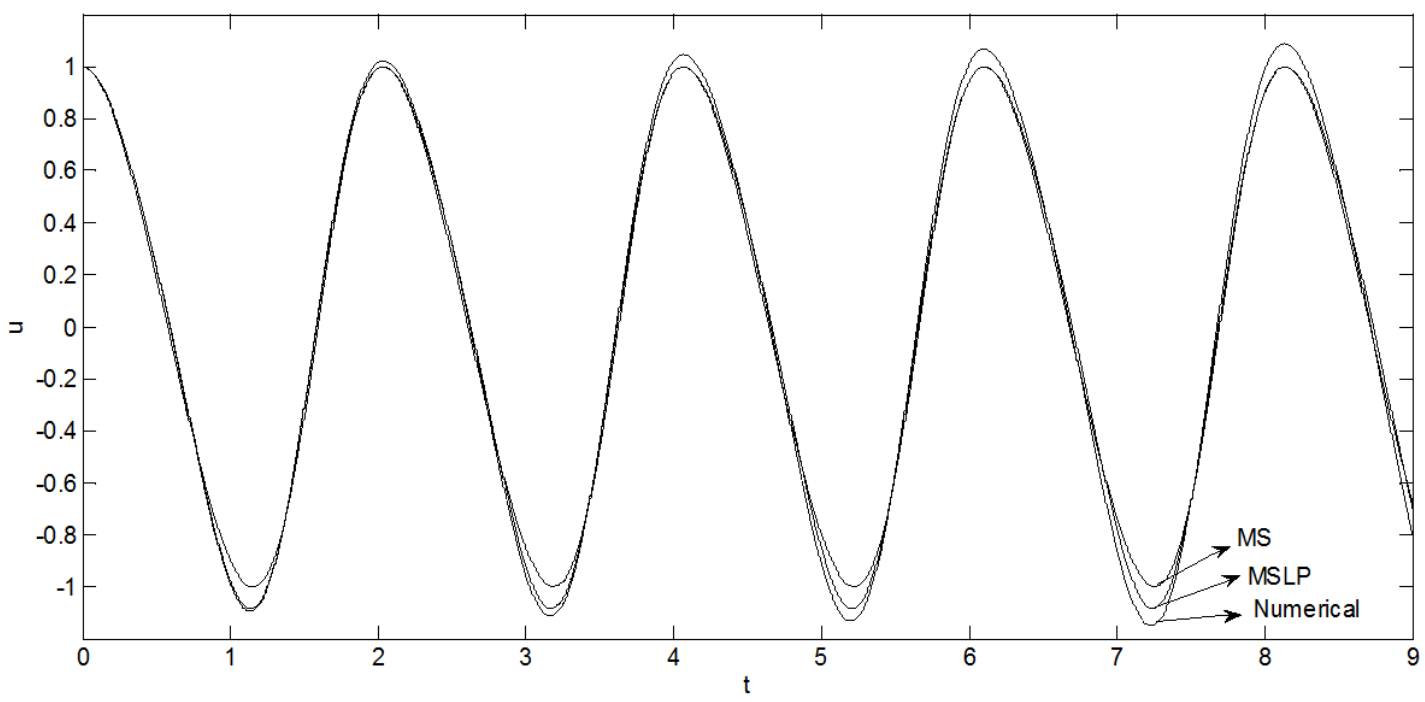

Figure 1. Comparison of numerical solutions and approximate analytical solutions (MS and MSLP) for $\varepsilon=2, a_{0}=1, \omega_{0}=\pi$ 


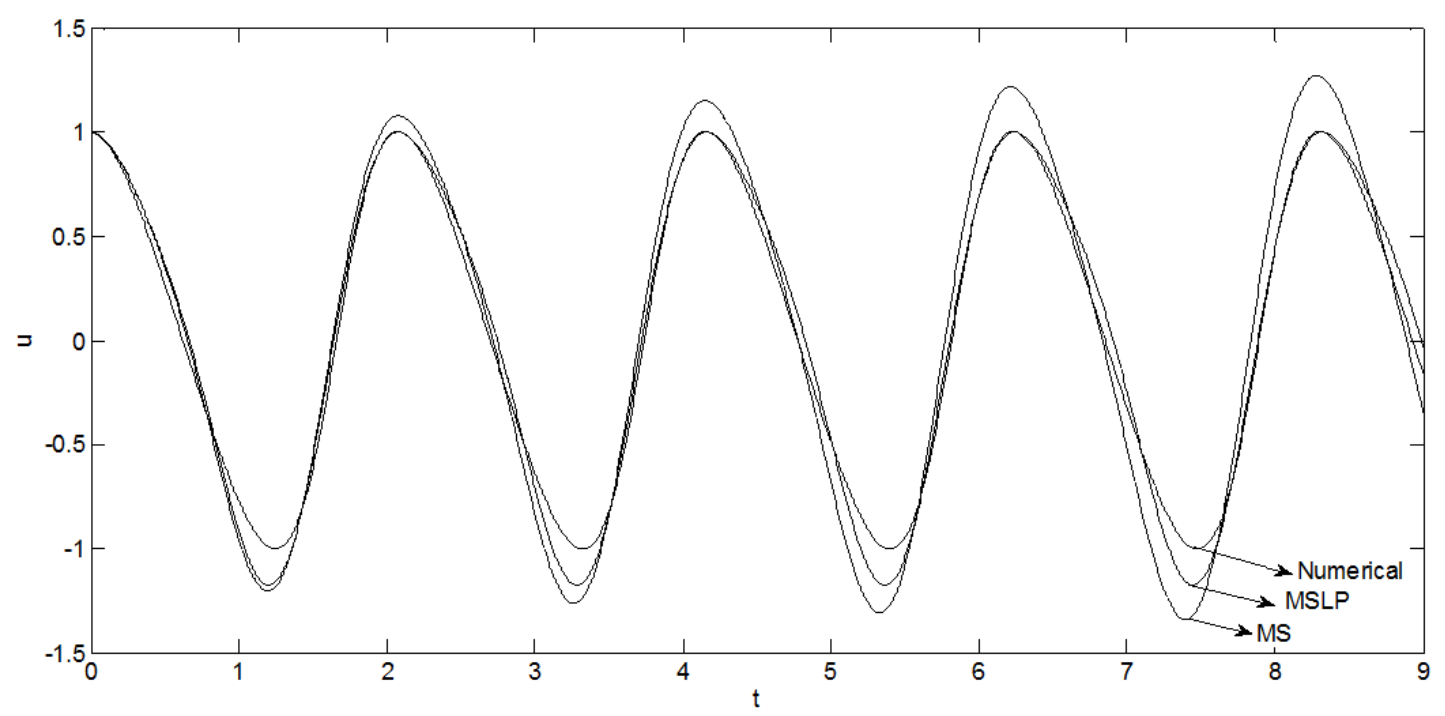

Figure 2. Comparison of numerical solutions and approximate analytical solutions (MS and MSLP) for $\varepsilon=3, a_{0}=1, \omega_{0}=\pi$

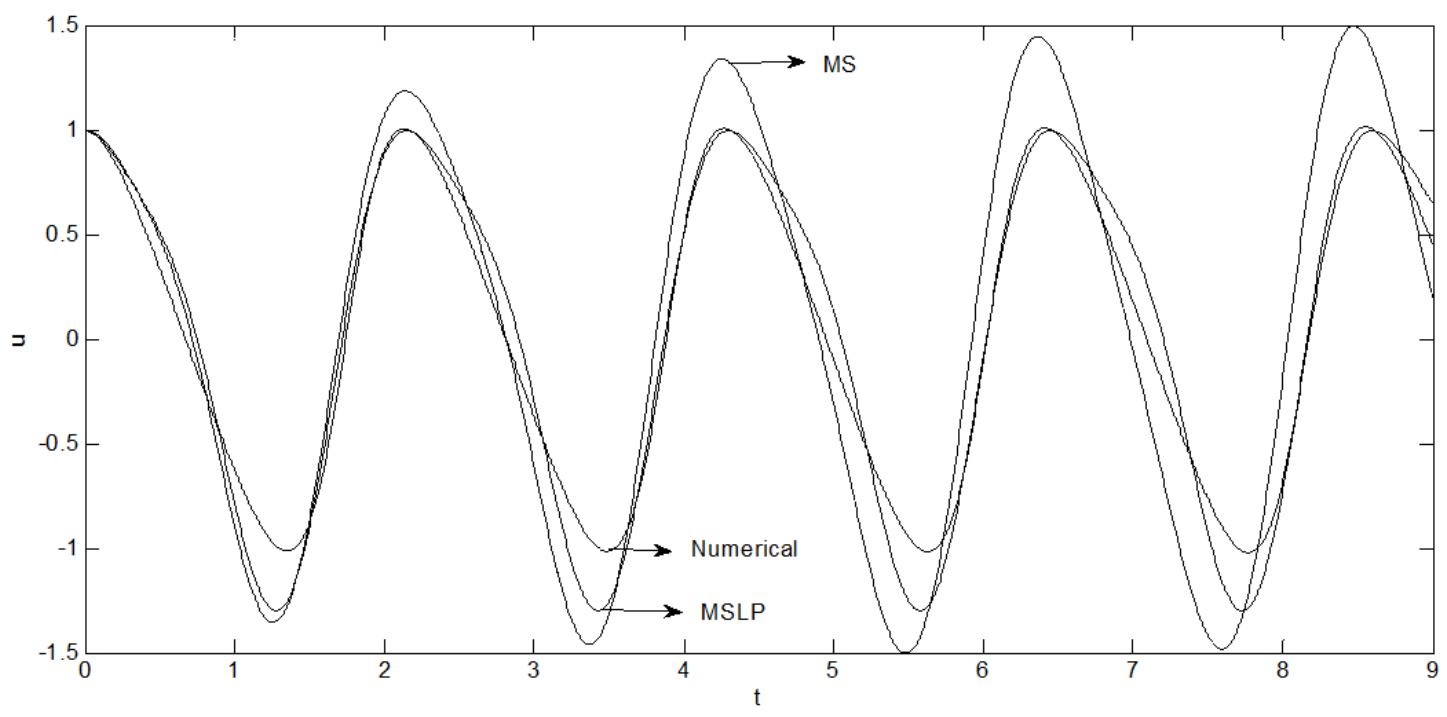

Figure 3. Comparison of numerical solutions and approximate analytical solutions (MS and MSLP) for $\varepsilon=4, a_{0}=1, \omega_{0}=\pi$

In this section, the approximate solutions are contrasted with the numerical solutions for the quadratic nonlinear model considered. In Figure 1, results are compared for $\varepsilon=2$. The agreement between MSLP and numerical solutions is better than MS solution and the amplitude values of the MS solution yield higher errors. The positive amplitudes agree with numerical and MSLP cases whereas the positive amplitudes introduce errors in case of MS solutions. For negative amplitude values, the error is less for MSLP. $\varepsilon=3$ is selected in Figure 2 and MSLP and numerical solutions agree well for positive values of amplitudes whereas, the error is smaller for negative amplitudes for MSLP solutions. For $\varepsilon=4$, in Figure 3, the same trend is observed with more amplification. 


\section{QUADRATIC NONLINEAR MODEL II}

Consider the below problem with a quadratic nonlinearity

$\ddot{u}+\omega_{0}^{2} u+\varepsilon \alpha u^{2}=0$

with initial conditions

$u(0)=a_{0} \quad \dot{u}(0)=0$

\subsection{Multiple Scales Method (MS)}

First, the problem is solved with the classical method. Solutions are assumed to be of the form;

$$
u=u_{0}\left(T_{0}, T_{1}, T_{2}\right)+\varepsilon u_{1}\left(T_{0}, T_{1}, T_{2}\right)+\varepsilon^{2} u_{2}\left(T_{0}, T_{1}, T_{2}\right)
$$

where $\mathrm{T}_{0}=\mathrm{t}$ is the usual fast time scale, $\mathrm{T}_{1}=\varepsilon \mathrm{t}$ and $\mathrm{T}_{2}=\varepsilon^{2} \mathrm{t}$ are the slow time scales. Time derivatives are defined as

$$
\frac{d}{d t}=D_{0}+\varepsilon D_{1}+\varepsilon^{2} D_{2}, \quad \frac{d^{2}}{d t^{2}}=D_{0}^{2}+2 \varepsilon D_{0} D_{1}+\varepsilon^{2}\left(D_{1}^{2}+2 D_{0} D_{2}\right)
$$

where $D_{n}=\partial / \partial T_{n}$.

If (42) and (43) are substituted into the original equation, the following equations are obtained at each order of $\varepsilon$;

$$
\begin{aligned}
& O(1) \quad D_{0}{ }^{2} u_{0}+\omega_{0}{ }^{2} u_{0}=0 \quad u_{0}(0)=a_{0} \quad D_{0} u_{0}(0)=0 \\
& \mathrm{O}(\varepsilon) \quad D_{0}{ }^{2} u_{1}+\omega_{0}{ }^{2} u_{1}=-2 D_{0} D_{1} u_{0}-\alpha u_{0}{ }^{2} \quad u_{1}(0)=0 \quad\left(D_{0} u_{1}+D_{1} u_{0}\right)(0)=0 \\
& \mathrm{O}\left(\varepsilon^{2}\right) \\
& D_{0}{ }^{2} u_{2}+\omega_{0}{ }^{2} u_{2}=-2 D_{0} D_{1} u_{1}-\left(D_{1}{ }^{2}+2 D_{0} D_{2}\right) u_{0}-2 \alpha u_{0} u_{1}
\end{aligned}
$$

At order 1, the solution may be expressed as

$$
u_{0}=A e^{i \omega_{0} T_{0}}+c c
$$

where cc stands for the complex conjugates of the preceding terms and

$$
A=\frac{1}{2} a e^{i \beta}
$$

The first order solution is obtained in terms of real amplitude and phase

$$
u_{0}=a\left(T_{1}, T_{2}\right) \cos \left(\omega_{0} T_{0}+\beta\left(T_{1}, T_{2}\right)\right)
$$

Applying the initial conditions yields

$$
\beta(0)=0 \quad a(0)=a_{0}
$$

Equation (47) is substituted into (45) and secular terms are eliminated

$$
D_{1} A=0 \Rightarrow A=A\left(T_{2}\right)
$$

The solution at order $\varepsilon$ is

$$
u_{1}=B e^{i \omega_{0} T_{0}}+c c+\frac{\alpha}{3 \omega_{0}{ }^{2}}\left(A^{2} e^{2 i \omega_{0} T_{0}}+c c\right)-\frac{\alpha}{\omega_{0}{ }^{2}} 2 A \bar{A}
$$

This solution may be represented in terms of real amplitude and phase

$$
u_{1}=b \cos \left(\omega_{0} T_{0}+\gamma\right)+\frac{\alpha}{6 \omega_{0}{ }^{2}} a^{2} \cos \left(2 \omega_{0} T_{0}+2 \beta\right)-\frac{\alpha}{2 \omega_{0}{ }^{2}} a^{2}
$$

where

$$
B=\frac{1}{2} i b e^{i \gamma}
$$

Applying the initial conditions yields

$$
\gamma(0)=0 \quad b(0)=\frac{\alpha}{3 \omega_{0}^{2}} a_{0}^{2}
$$


Equation (47) and (52) are inserted into (46) and secular terms are eliminated,

$$
-2 i \omega_{0} D_{1} B-2 i \omega_{0} D_{2} A+\frac{10}{3} \frac{\alpha^{2}}{\omega_{0}^{2}} A^{2} \bar{A}=0
$$

If (45), (54), (55) are inserted into (56), one finally has

$$
a=a_{0}, \quad b=\frac{\alpha}{3 \omega_{0}^{2}} a_{0}^{2}, \quad \gamma=0, \quad \beta=-\frac{5}{12} \frac{\alpha^{2}}{\omega_{0}^{3}} a_{0}^{2} T_{2}
$$

The final solution is

$$
\begin{aligned}
& u=a_{0} \cos \left(\left(\omega_{0}-\varepsilon^{2} \frac{5 \alpha^{2}}{12 \omega_{0}{ }^{3}} a_{0}{ }^{2}\right) t\right)+\varepsilon\left(\frac{\alpha}{3 \omega_{0}{ }^{2}} a_{0}{ }^{2} \cos \left(\omega_{0} t\right)\right. \\
& +\frac{\alpha}{6 \omega_{0}{ }^{2}} a_{0}{ }^{2}\left(\cos \left(2\left(\omega_{0}-\varepsilon^{2} \frac{5 \alpha^{2}}{12 \omega_{0}{ }^{3}} a_{0}{ }^{2}\right) t\right)-3\right)+O\left(\varepsilon^{2}\right)
\end{aligned}
$$

\subsection{Multiple Scales Lindstedt-Poincare Method (MSLP)}

The time transformation $\tau=\omega t$ is applied to the model

$$
\omega^{2} u^{\prime \prime}+\omega_{0}{ }^{2} u+\varepsilon \alpha u^{2}=0
$$

where prime denotes derivative with respect to the new variable $\tau$. The time scales in this method are slightly different from classical multiple scales

$$
T_{0}=\tau, T_{1}=\varepsilon \tau, T_{2}=\varepsilon^{2} \tau
$$

Expressing the time derivatives

$$
\frac{d}{d t}=D_{0}+\varepsilon D_{1}+\varepsilon^{2} D_{2} \quad \frac{d^{2}}{d t^{2}}=D_{0}^{2}+2 \varepsilon D_{0} D_{1}+\varepsilon^{2}\left(D_{1}^{2}+2 D_{0} D_{2}\right)
$$

with $\mathrm{D}_{\mathrm{n}}=\partial / \partial T_{n}$ and substituting the expansions

$$
\begin{aligned}
& u=u_{0}\left(T_{0}, T_{1}, T_{2}\right)+\varepsilon u_{1}\left(T_{0}, T_{1}, T_{2}\right)+\varepsilon^{2} u_{2}\left(T_{0}, T_{1}, T_{2}\right) \\
& \omega_{0}{ }^{2}=\omega^{2}-\varepsilon \omega_{1}-\varepsilon^{2} \omega_{2}
\end{aligned}
$$

into the original equation, the following equations are obtained at each order of approximation;

$$
\begin{aligned}
& O(1) \quad \omega^{2} D_{0}{ }^{2} u_{0}+\omega^{2} u_{0}=0, \quad u_{0}(0)=a_{0} \quad D_{0} u_{0}(0)=0 \\
& \mathrm{O}(\varepsilon) \quad \omega^{2} D_{0}{ }^{2} u_{1}+\omega^{2} u_{1}=-2 \omega^{2} D_{0} D_{1} u_{0}+\omega_{1} u_{0}-\alpha u_{0}{ }^{2} \quad u_{1}(0)=0 \quad\left(D_{0} u_{1}+D_{1} u_{0}\right)(0)=0 \\
& \mathrm{O}\left(\varepsilon^{2}\right) \quad \omega^{2} D_{0}{ }^{2} u_{2}+\omega^{2} u_{2}=-2 \omega^{2} D_{0} D_{1} u_{1}-\omega^{2}\left(D_{1}{ }^{2}+2 D_{0} D_{2}\right) u_{0}+\omega_{1} u_{1}+\omega_{2} u_{0}-2 \alpha u_{0} u_{1}
\end{aligned}
$$

The first order solution is

$$
u_{0}=A e^{i T_{0}}+c c=a \cos \left(T_{0}+\beta\right)
$$

Applying the initial conditions yields

$$
\beta(0)=0, a(0)=a_{0}
$$

Equation (67) is substituted into (65) and secular terms are eliminated

$$
-2 i \omega^{2} D_{1} A+\omega_{1} A=0
$$

If $D_{1} A=0$ is selected, $a=a\left(T_{2}\right), \beta=\beta\left(T_{2}\right)$ and $\omega_{1}=0$. Since $\omega_{1}$ is not complex, this choice is admissible. The solution at order $\varepsilon$ is

$$
u_{1}=B e^{i T_{0}}+c c+\frac{\alpha}{3 \omega^{2}}\left(A^{2} e^{2 i T_{0}}+c c\right)-\frac{\alpha}{\omega^{2}} 2 A \bar{A} \quad B=\frac{1}{2} i b e^{i \gamma}
$$

In terms of real amplitude and phase, the solution is

$$
u_{1}=b \cos \left(T_{0}+\gamma\right)+\frac{\alpha}{6 \omega^{2}} a^{2} \cos \left(2 T_{0}+2 \beta\right)-\frac{\alpha}{2 \omega^{2}} a^{2}
$$

Applying the initial conditions yields 


$$
\gamma(0)=0 \quad b(0)=\frac{\alpha}{3 \omega^{2}} a_{0}^{2}
$$

Equations (67) and (70) are inserted into (66) and secular terms are eliminated

$$
-2 i \omega^{2} D_{1} B-2 i \omega^{2} D_{2} A+\omega_{2} A+\left(4-\frac{2}{3}\right) \frac{\alpha^{2}}{\omega^{2}} A^{2} \bar{A}=0
$$

$\mathrm{D}_{1} \mathrm{~B}=0$ can be assumed. If $\mathrm{D}_{2} \mathrm{~A}=0$ is selected, $\omega_{2}$ comes out to be real and this is again an admissible choice. After algebraic calculations, Equation (73) yields

$$
a=a_{0}, \quad b=\frac{\alpha}{3 \omega^{2}} a_{0}^{2}, \quad \gamma=\beta=0, \quad \omega_{2}=-\frac{5}{6} \frac{\alpha^{2}}{\omega^{2}} a^{2}
$$

The frequency is

$$
\omega=\sqrt{\frac{1}{2} \omega_{0}^{2}+\frac{1}{2} \sqrt{\omega_{0}^{4}-\frac{10}{3} \varepsilon^{2} \alpha^{2} a_{0}^{2}}}
$$

The final solution in terms of this frequency is

$$
u=a_{0} \cos (\omega t)+\varepsilon\left(\frac{\alpha}{3 \omega^{2}} a_{0}{ }^{2} \cos (\omega t)+\frac{\alpha a_{0}{ }^{2}}{6 \omega^{2}}(\cos (2 \omega t)-3)\right)+O\left(\varepsilon^{2}\right)
$$

For valid solutions, the perturbation criteria is

$$
\frac{\varepsilon \alpha a_{0}}{3 \omega^{2}}<<1
$$

\subsection{Comparisons with the Numerical Solutions}

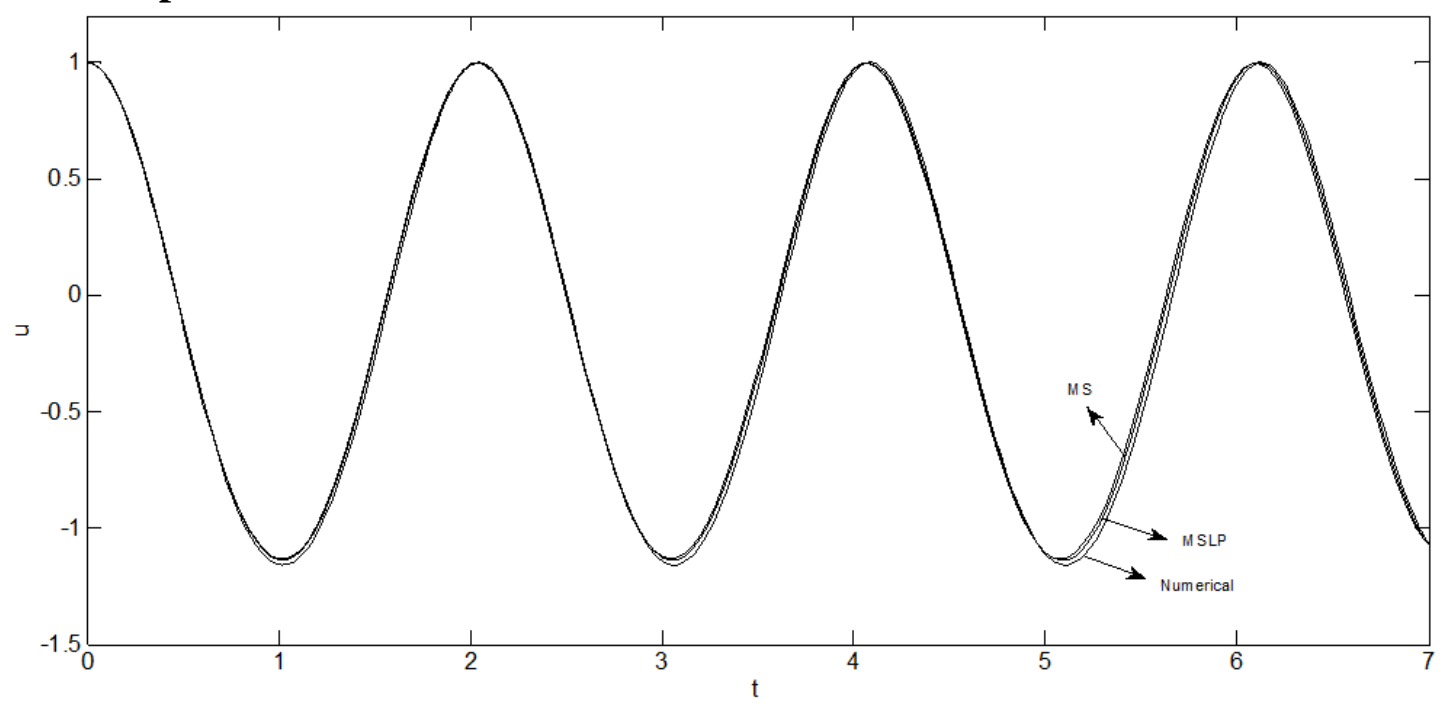

Figure 4. Comparison of numerical solutions and approximate analytical solutions

(MS and MSLP) for $\varepsilon=2, a_{0}=1, \omega_{0}=\pi$ 


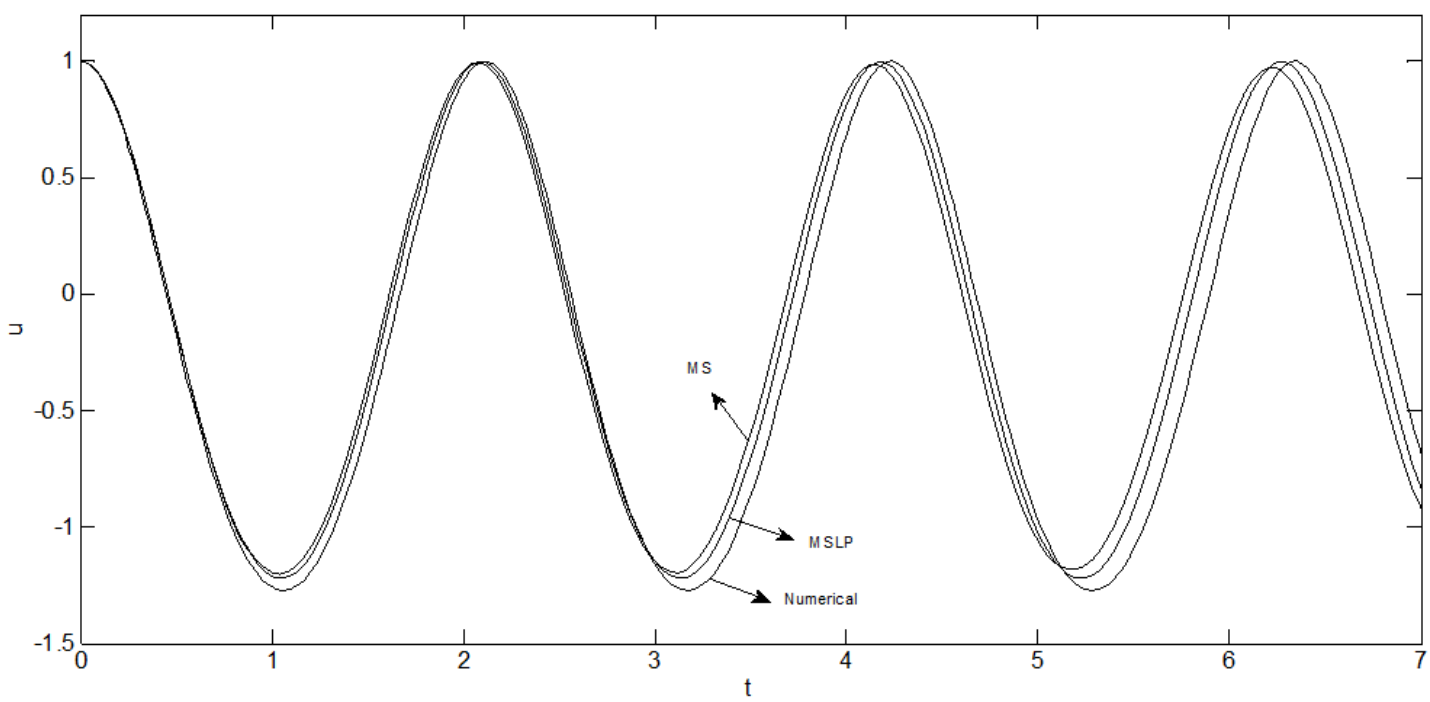

Figure 5. Comparison of numerical solutions and approximate analytical solutions (MS and MSLP) for $\varepsilon=3, a_{0}=1, \omega_{0}=\pi$

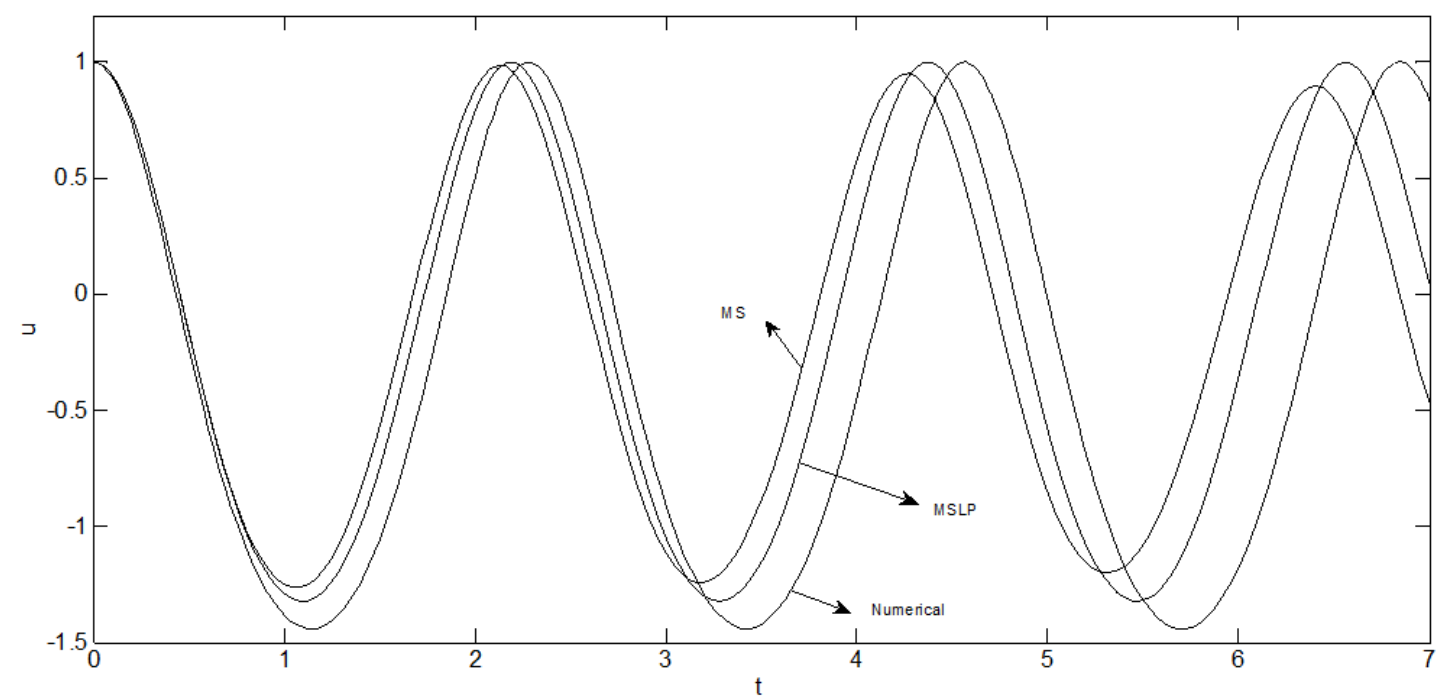

Figure 6. Comparison of numerical solutions and approximate analytical solutions (MS and MSLP) for $\varepsilon=4, a_{0}=1, \omega_{0}=\pi$

In this section, the approximate solutions are contrasted with the numerical solutions for the quadratic nonlinear model considered. In Figure 4, results are compared for $\varepsilon=2$. The agreement between MSLP and numerical solutions is better than MS solution and the amplitude values of the MS solution yield higher errors. $\varepsilon=3$ is selected in Figure 5 and $\varepsilon=4$ is selected in Figure 6 . In Figures 5 and 6 , the same trend is observed with more amplification. 


\section{QUADRATIC NONLINERITY WITH DAMPING}

A damping term is added to the previous model

$\ddot{u}+\omega_{0}^{2} u+2 \varepsilon \mu \dot{u}+\varepsilon \alpha u^{2}=0$

with initial conditions

$u(0)=a_{0} \quad \dot{u}(0)=0$

\subsection{Multiple Scales Method (MS)}

First, the problem is solved with the classical method. Solutions are assumed to be of the form;

$$
u=u_{0}\left(T_{0}, T_{1}, T_{2}\right)+\varepsilon u_{1}\left(T_{0}, T_{1}, T_{2}\right)+\varepsilon^{2} u_{2}\left(T_{0}, T_{1}, T_{2}\right)
$$

where $\mathrm{T}_{0}=\mathrm{t}$ is the usual fast time scale, $\mathrm{T}_{1}=\varepsilon \mathrm{t}$ and $\mathrm{T}_{2}=\varepsilon^{2} \mathrm{t}$ are the slow time scales. Time derivatives are defined as

$$
\frac{d}{d t}=D_{0}+\varepsilon D_{1}+\varepsilon^{2} D_{2}, \quad \frac{d^{2}}{d t^{2}}=D_{0}^{2}+2 \varepsilon D_{0} D_{1}+\varepsilon^{2}\left(D_{1}^{2}+2 D_{0} D_{2}\right)
$$

where $D_{n}=\partial / \partial T_{n}$.

If (80) and (81) are substituted into the original equation, the following equations are obtained at each order of $\varepsilon$;

$$
\begin{aligned}
& O(1) \quad D_{0}{ }^{2} u_{0}+\omega_{0}{ }^{2} u_{0}=0 \quad u_{0}(0)=a_{0} \quad D_{0} u_{0}(0)=0 \\
& \mathrm{O}(\varepsilon) \quad D_{0}{ }^{2} u_{1}+\omega_{0}{ }^{2} u_{1}=-2 D_{0} D_{1} u_{0}-2 \mu D_{0} u_{0}-\alpha u_{0}^{2} \quad u_{1}(0)=0 \quad\left(D_{0} u_{1}+D_{1} u_{0}\right)(0)=0 \\
& \mathrm{O}\left(\varepsilon^{2}\right) \quad D_{0}^{2} u_{2}+\omega_{0}^{2} u_{2}=-2 D_{0} D_{1} u_{1}-\left(D_{1}^{2}+2 D_{0} D_{2}\right) u_{0}-2 \mu\left(D_{0} u_{1}+D_{1} u_{0}\right)-2 \alpha u_{0} u_{1}
\end{aligned}
$$

At order 1, the solution may be expressed as

$u_{0}=A e^{i \omega_{0} T_{0}}+c c$

where cc stands for the complex conjugates of the preceding terms and

$$
A=\frac{1}{2} a e^{i \beta}
$$

The first order solution is obtained in terms of real amplitude and phase

$$
u_{0}=a\left(T_{1}, T_{2}\right) \cos \left(\omega_{0} T_{0}+\beta\left(T_{1}, T_{2}\right)\right)
$$

Applying the initial conditions yields

$$
\beta(0)=0 \quad a(0)=a_{0}
$$

Equation (85) is substituted into (83) and secular terms are eliminated

$$
D_{1} A=-\mu A \Rightarrow a=a\left(T_{2}\right) e^{-\mu T_{1}}, \quad \beta=\beta\left(T_{2}\right)
$$

The solution at order $\varepsilon$ is

$$
u_{1}=B e^{i \omega_{0} T_{0}}+c c+\frac{\alpha}{3 \omega_{0}{ }^{2}}\left(A^{2} e^{2 i \omega_{0} T_{0}}+c c\right)-\frac{\alpha}{\omega_{0}{ }^{2}} 2 A \bar{A}
$$

This solution may be represented in terms of real amplitude and phase

$$
u_{1}=b \cos \left(\omega_{0} T_{0}+\gamma\right)+\frac{\alpha}{6 \omega_{0}{ }^{2}} a^{2} \cos \left(2 \omega_{0} T_{0}+2 \beta\right)-\frac{\alpha}{2 \omega_{0}{ }^{2}} a^{2}
$$

where

$$
B=\frac{1}{2} i b e^{i \gamma}
$$

Applying the initial conditions yields

$$
\gamma(0)=\operatorname{Arctan}\left(-\frac{3 \mu \omega_{0}}{\alpha a_{0}}\right), \quad b(0)=\frac{a_{0}}{\omega_{0}} \sqrt{\frac{\alpha^{2} a_{0}{ }^{2}}{9 \omega_{0}{ }^{2}}+\mu^{2}}
$$


Equation (85) and (90) are inserted into (84) and secular terms are eliminated,

$$
-2 i \omega_{0} D_{1} B-D_{1}^{2} A-2 i \omega_{0} D_{2} A-2 \mu i \omega_{0} B-2 \mu D_{1} A+\frac{\alpha^{2}}{\omega_{0}{ }^{2}}\left(4-\frac{2}{3}\right) A^{2} \bar{A}=0
$$

If (82), (92), (93) are inserted into (94), one finally has

$$
a=a_{0} e^{-\mu T_{1}}, \quad b=b_{0} e^{-\mu T_{1}}, \quad \gamma=\gamma_{0}, \quad \beta=-\left(\frac{\mu^{2}}{2 \omega_{0}}+\frac{5 \alpha^{2}}{12 \omega_{0}^{3}} a^{2}\right) T_{2}
$$

The final solution is

$$
\begin{aligned}
& u=a_{0} e^{-\varepsilon \mu t} \cos \left(\left(\omega_{0}-\varepsilon^{2}\left(\frac{\mu^{2}}{2 \omega_{0}}+\frac{5}{12} \frac{\alpha^{2}}{\omega_{0}^{3}} a_{0}^{2} e^{-2 \varepsilon \mu t}\right)\right) t\right) \\
& +\varepsilon\left(\frac{a_{0}}{\omega_{0}} \sqrt{\frac{\alpha^{2} a_{0}^{2}}{9 \omega_{0}^{2}}+\mu^{2}} e^{-\varepsilon \mu t} \cos \left(\omega_{0} t+\operatorname{Arctan}\left(\frac{-3 \mu \omega_{0}}{\alpha a_{0}}\right)\right)\right. \\
& +\frac{\alpha}{6 \omega_{0}^{2}} a_{0}^{2} e^{-2 \varepsilon \mu t}\left(\cos \left(2\left(\omega_{0}-\varepsilon^{2}\left(\frac{\mu^{2}}{2 \omega_{0}}+\frac{5}{12} \frac{\alpha^{2}}{\omega_{0}^{3}} a_{0}^{2} e^{-2 \varepsilon \mu t}\right)\right) t\right)-3\right)+O\left(\varepsilon^{2}\right)
\end{aligned}
$$

\subsection{Multiple Scales Lindstedt-Poincare Method (MSLP)}

The time transformation $\tau=\omega t$ is applied to the model

$$
\omega^{2} u^{\prime \prime}+\omega_{0}^{2} u+2 \varepsilon \mu \omega u^{\prime}+\varepsilon \alpha u^{2}=0
$$

where prime denotes derivative with respect to the new variable $\tau$. The time scales in this method are slightly different from classical multiple scales

$$
T_{0}=\tau, T_{1}=\varepsilon \tau, T_{2}=\varepsilon^{2} \tau
$$

Expressing the time derivatives

$$
\frac{d}{d t}=D_{0}+\varepsilon D_{1}+\varepsilon^{2} D_{2} \quad \frac{d^{2}}{d t^{2}}=D_{0}^{2}+2 \varepsilon D_{0} D_{1}+\varepsilon^{2}\left(D_{1}^{2}+2 D_{0} D_{2}\right)
$$

with $\mathrm{D}_{\mathrm{n}}=\partial / \partial T_{n}$ and substituting the expansions

$$
\begin{aligned}
& u=u_{0}\left(T_{0}, T_{1}, T_{2}\right)+\varepsilon u_{1}\left(T_{0}, T_{1}, T_{2}\right)+\varepsilon^{2} u_{2}\left(T_{0}, T_{1}, T_{2}\right) \\
& \omega_{0}{ }^{2}=\omega^{2}-\varepsilon \omega_{1}-\varepsilon^{2} \omega_{2}
\end{aligned}
$$

into the original equation, the following equations are obtained at each order of approximation;

$$
\begin{aligned}
& O(1) \quad \omega^{2} D_{0}^{2} u_{0}+\omega^{2} u_{0}=0, \quad u_{0}(0)=a_{0} \quad D_{0} u_{0}(0)=0 \\
& \mathrm{O}(\varepsilon) \quad \omega^{2} D_{0}^{2} u_{1}+\omega^{2} u_{1}=-2 \omega^{2} D_{0} D_{1} u_{0}+\omega_{1} u_{0}-\alpha u_{0}^{2}-2 \mu \omega D_{0} u_{0} \quad u_{1}(0)=0 \quad\left(D_{0} u_{1}+D_{1} u_{0}\right)(0)=0 \\
& \mathrm{O}\left(\varepsilon^{2}\right) \quad \omega^{2} D_{0}{ }^{2} u_{2}+\omega^{2} u_{2}=-2 \omega^{2} D_{0} D_{1} u_{1}-\omega^{2}\left(D_{1}^{2}+2 D_{0} D_{2}\right) u_{0}+\omega_{1} u_{1}+\omega_{2} u_{0}-2 \alpha u_{0} u_{1}-2 \mu \omega\left(D_{0} u_{1}+D_{1} u_{0}\right)
\end{aligned}
$$

The first order solution is

$$
u_{0}=A e^{i T_{0}}+c c=a \cos \left(T_{0}+\beta\right)
$$

Applying the initial conditions yields

$$
\beta(0)=0, a(0)=a_{0}
$$

Equation (105) is substituted into (103) and secular terms are eliminated

$$
-2 i \omega^{2} D_{1} A+\omega_{1} A-2 \mu \omega i A=0
$$

If $D_{1} A=-\frac{\mu}{\omega} A$ is selected, $a=a\left(T_{2}\right) e^{-\frac{\mu}{\omega} T_{1}}, \beta=\beta\left(\mathrm{T}_{2}\right)$ and $\omega_{1}=0$. Since $\omega_{1}$ is not complex, this choice is admissible. The solution at order $\varepsilon$ is

$$
u_{1}=B e^{i T_{0}}+c c+\frac{\alpha}{3 \omega^{2}}\left(A^{2} e^{2 i T_{0}}+c c\right)-\frac{\alpha}{\omega^{2}} 2 A \bar{A} \quad B=\frac{1}{2} i b e^{i \gamma}
$$


In terms of real amplitude and phase, the solution is

$$
u_{1}=b \cos \left(T_{0}+\gamma\right)+\frac{\alpha}{6 \omega^{2}} a^{2} \cos \left(2 T_{0}+2 \beta\right)-\frac{\alpha}{2 \omega^{2}} a^{2}
$$

Applying the initial conditions yields

$$
\gamma(0)=\operatorname{Arctan}\left(-\frac{3 \mu \omega}{\alpha a_{0}}\right), b(0)=\sqrt{\frac{\alpha^{2}}{9 \omega^{2}} a_{0}^{2}+\mu^{2}} \frac{a_{0}}{\omega}
$$

Equations (105) and (108) are inserted into (104) and secular terms are eliminated

$$
-2 i \omega^{2} D_{1} B-2 i \omega^{2} D_{2} A+\omega_{2} A+\left(\frac{10}{3}\right) \frac{\alpha^{2}}{\omega^{2}} A^{2} \bar{A}=0
$$

$D_{1} B=-\frac{\mu}{\omega} B$ can be assumed. If $\mathrm{D}_{2} \mathrm{~A}=0$ is selected, $\omega_{2}$ comes out to be real and this is again an admissible choice. After algebraic calculations, Equation (111) yields

$a=a_{0} e^{-\varepsilon \mu t}, \quad b=\sqrt{\frac{\alpha^{2}}{9 \omega^{2}} a_{0}^{2}+\mu^{2}} \frac{a_{0}}{\omega} e^{-\frac{\mu}{\omega} T_{1}}, \quad \gamma=\gamma_{0}=\operatorname{Arctan}\left(-\frac{3 \mu \omega}{\alpha a_{0}}\right), \quad \beta=\beta_{0}=0, \quad \omega_{2}=-\frac{5}{6} \frac{\alpha^{2}}{\omega^{2}} a^{2}-\mu^{2}$

The frequency is

$$
\omega=\sqrt{\frac{1}{2}\left(\omega_{0}^{2}-\varepsilon^{2} \mu^{2}\right)+\frac{1}{2} \sqrt{\left(\omega_{0}^{2}-\varepsilon^{2} \mu^{2}\right)^{2}-\frac{10}{3} \varepsilon^{2} \alpha^{2} a^{2}}}
$$

The final solution in terms of this frequency is

$$
\begin{aligned}
& u=a_{0} e^{-\varepsilon \mu t} \cos (\omega t)+\varepsilon\left(\frac{a_{0}}{\omega} \sqrt{\frac{\alpha^{2}}{9 \omega^{2}} a_{0}^{2}+\mu^{2}} e^{-\varepsilon \mu t} \cos \left(\omega t+\tan ^{-1}\left(-\frac{3 \mu \omega}{\alpha a_{0}}\right)\right)\right. \\
& \left.+\frac{\alpha a_{0}^{2}}{6 \omega^{2}} e^{-2 \varepsilon \mu t}(\cos (2 \omega t)-3)\right)+O\left(\varepsilon^{2}\right)
\end{aligned}
$$

For valid solutions, the perturbation criteria is

$$
\frac{\varepsilon \alpha a}{6 \omega^{2}}<<1
$$

\subsection{Comparisons with the Numerical Solutions}

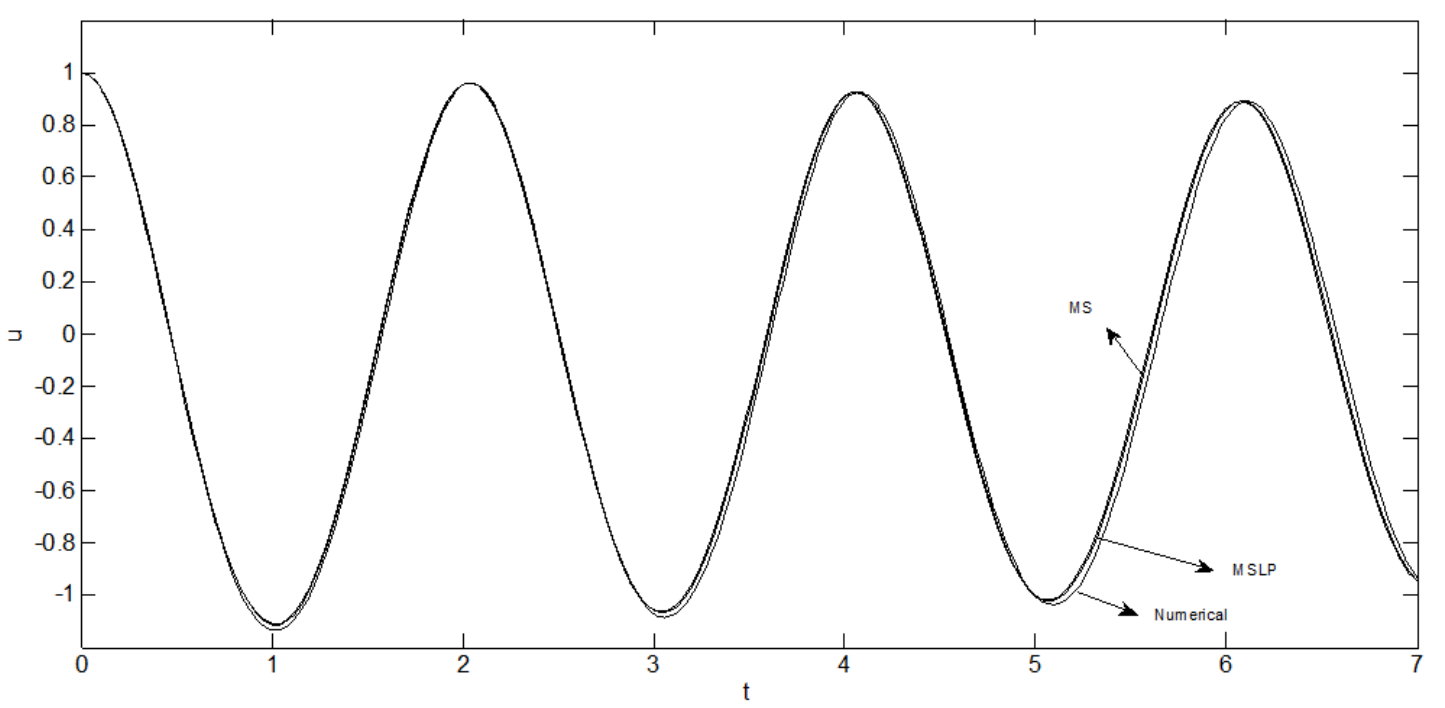

Figure 7. Comparison of numerical solutions and approximate analytical solutions (MS and MSLP) for $\varepsilon=2, a_{0}=1, \omega_{0}=\pi, \mu=0.01$ 


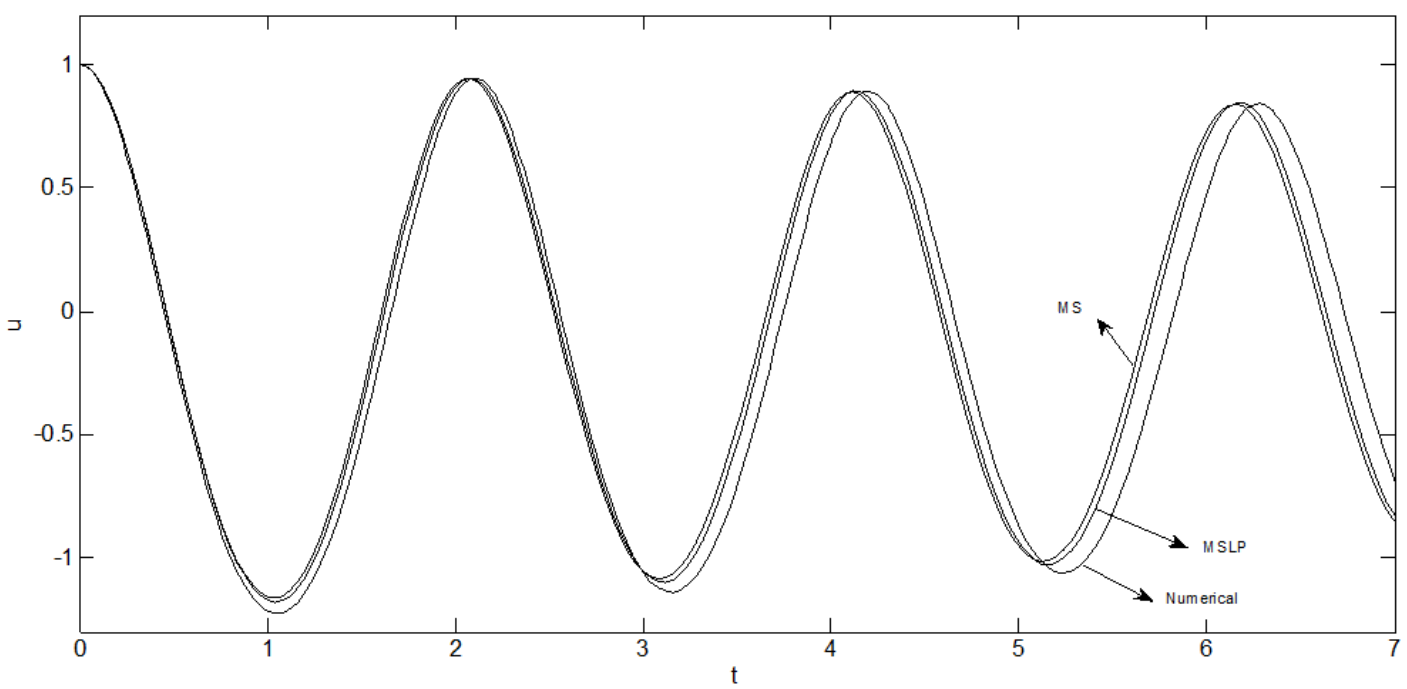

Figure 8. Comparison of numerical solutions and approximate analytical solutions (MS and MSLP) for $\varepsilon=3, a_{0}=1, \omega_{0}=\pi, \mu=0.01$

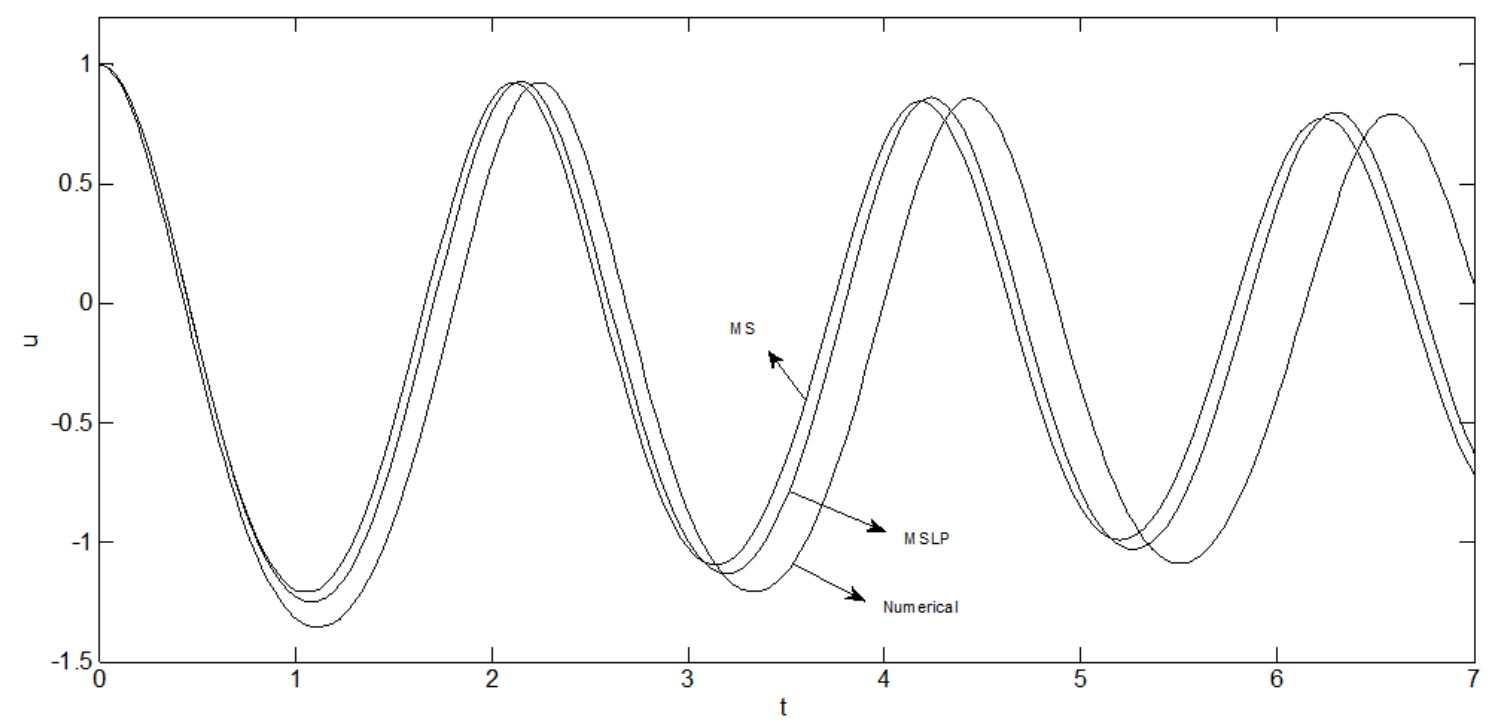

Figure 9. Comparison of numerical solutions and approximate analytical solutions (MS and MSLP) for $\varepsilon=4, a_{0}=1, \omega_{0}=\pi, \mu=0.01$

The approximate solutions are contrasted with the numerical solutions for the quadratic nonlinear model with damping. In Figure 7, results are compared for $\varepsilon=2$. The agreement between MSLP and numerical solutions is better than MS solution and the amplitude values of the MS solution yield higher errors. $\varepsilon=3$ is selected in Figure 8 and $\varepsilon=4$ is selected in Figure 9. In summary, the MSLP solutions are better compared to the MS solutions. 


\section{CONCLUDING REMARKS}

A recently developed perturbation technique namely the multiple scales Lindstedt Poincare method (MSLP) combining the multiple scales and Lindstedt Poincare methods is applied to three different mathematical models with quadratic nonlinearities.

In conclusion, the MSLP method yields closer solutions to the numerical ones compared to those of MS for strong quadratic nonlinearities.

\section{REFERENCES}

1. M. Pakdemirli, M.M.F. Karahan, H. Boyac1, A new perturbation algorithm with better convergence properties: Multiple Scales Lindstedt-Poincare Method. Mathematical and Computational Applications 14(1), 31-44, 2009.

2. M. Pakdemirli and M. M. F. Karahan, A new perturbation solution for systems with strong quadratic and cubic nonlinearities, Mathematical Methods in the Applied Sciences 3, 704-712, 2010.

3. M. Pakdemirli, M. M. F. Karahan and H. Boyac1, Forced Vibrations of Strongly Nonlinear Systems with Multiple Scales Lindstedt Poicare Method, Mathematical and Computational Applications 16(4), 879-889, 2011. 